\title{
Adaptive expression of biofilm regulators and adhesion factors of Staphylococcus aureus during acute wound infection under the treatment of negative pressure wound therapy in vivo
}

\author{
TONGTONG LI ${ }^{1 *}$, GUOQI WANG ${ }^{2 *}$, PENG YIN ${ }^{3 *}$, ZHIRUI LI $^{4}$, LIHAI ZHANG $^{4}$ and PEIFU TANG ${ }^{4}$ \\ ${ }^{1}$ Department of Orthopedics, Tianjin Hospital, Tianjin 300211; ${ }^{2}$ Department of Pediatrics, Chinese People's Liberation \\ Army (PLA) General Hospital, Beijing 100853; ${ }^{3}$ Department of Orthopedics, Beijing Chao-Yang Hospital, Beijing 100043; \\ ${ }^{4}$ Department of Orthopedics, Chinese People's Liberation Army (PLA) General Hospital, Beijing 100853, P.R. China
}

Received September 24, 2019; Accepted March 17, 2020

DOI: 10.3892/etm.2020.8679

\begin{abstract}
Negative pressure wound therapy (NPWT) is gaining acceptance as a physical therapy for a wide variety of infected wounds. To gain insight into the response of bacteria to NPWT in vivo, the adaptive expression of biofilm regulators and adhesion factors of Staphylococcus aureus (S. aureus), the most frequently isolated pathogen in the clinic, during acute wound infection was investigated. A $3 \mathrm{~cm}$ full-thickness dermal wound was created on each side of a rabbit back and inoculated with green fluorescent protein-labeled $S$. aureus. NPWT was initiated at $6 \mathrm{~h}$ post inoculation, with the wound on the contralateral side as the untreated self-control. The wounds were subjected to a 28 day observation period. Histological analysis, laser scanning confocal microscopy and scanning electron microscopy revealed a transition of $S$. aureus to a free-living phenotype in tissues treated with NPWT, compared with microcolonies in untreated wounds. Viable bacteria counts showed a modest reduction in the bioburden of NPWT group on day $8(\mathrm{P}<0.001)$, with $\sim 1 \times 10^{6}$ colony-forming units/g tissue. Transcript analysis of biofilmand colonization-related genes were investigated using reverse transcription-quantitative PCR on postoperative days 1, 2, 4 and 8 . The poly-beta-1,6-N-acetyl-D-glucosamine synthase locus and holin-like protein CidA/antiholin-like protein $\operatorname{Lrg} \mathrm{A}$ network were less active in the NPWT group compared with the untreated control group. Accordingly, the expression profile switched to an elevated expression of the adhesive factors UDP-phosphate N-acetylglucosaminyl 1-phosphate
\end{abstract}

Correspondence to: Dr Peifu Tang, Department of Orthopedics, Chinese People's Liberation Army (PLA) General Hospital, 28 Fuxing Road, Beijing 100853, P.R. China

E-mail: tangpeifu301@sina.cn

${ }^{*}$ Contributed equally

Key words: negative pressure wound therapy, biofilm, bacterial colonization, Staphylococcus aureus, acute wound transferase (at days 0-4) and fibronectin-binding protein A and iron-regulated surface determinant protein $\mathrm{A}$ at $>4$ days during both stages of colonization. Meanwhile, low expression levels of the effector molecule (RNAIII) of the accessory gene regulator type I (agr) system was detected in NPWT group, suggesting that the bacterial density in NPWT-treated wounds was under the threshold for agr activation, thus not leading to an active and invasive infection. The wounds treated by NPWT healed completely on day 28 , compared with an average of an $8.11 \%$ defect area in the control group $(\mathrm{P}<0.001)$. The results of the current study indicated that $S$. aureus responds to NPWT by regulating gene expression, manifesting a decrease in biofilm formation and an increase in bacterial colonization in vivo, which potentially benefits the wound repair and healing process.

\section{Introduction}

As the predominant pathogen in both community- and hospital-acquired wound infections, Staphylococcus aureus (S. aureus) has attracted widespread attention and has been extensively studied (1). Bacterial colonization, which is defined as the presence of replicating microorganisms adherent to the wound in the absence of tissue damage, is the initial step in the development of wound infection (2). A variety of surface-associated factors are involved in the attachment and colonization of $S$. aureus to host cells and extracellular matrix, such as wall teichoic acid (WTA), fibronectin-binding protein A/B (FnBPA/B), iron-regulated surface determinant A (IsdA), clumping factor $\mathrm{A} / \mathrm{B}$ and collagen-binding protein $(2,3)$. In particular, WTA (a surface-exposed polyanionic polymer of the cell wall), which is biosynthesized by the enzyme UDP-phosphate N-acetylglucosaminyl 1-phosphate transferase $(\operatorname{tag} O)$, serves a crucial role in the initiation of $S$. aureus colonization (4). FnBPA and IsdA, which are members of the microbial surface components recognizing adhesive matrix molecules protein family and encoded by $f n b A$ and $i s d A$, respectively, serve a leading role in prolonged persistence in wounds $(2,5)$.

Bacterial colonization alone was reported to exhibit little harm to host cells and the wound healing process $(6,7)$. 
However, colonized bacteria can form complex microbial communities, which are called biofilms, and embed themselves in extracellular polymeric substances (EPS), serving as a shield against host immune cells and antibacterial agents (8). Polysaccharide intercellular adhesion (synthesized by the enzyme encoded by the ica operon) and extracellular DNA [cooperatively regulated by the holin-like protein CidA (cidA)/antiholin-like protein $\operatorname{LrgA}(\operatorname{lrg} A)$ network and LysR family regulatory protein CidR (cidR)] are the main components of EPS, exhibiting an important effect on intercellular adhesion and biofilm formation in vitro and in vivo $(8,9)$. With the protection of the biofilm matrix, $S$. aureus proliferates continually to a critical cell density, at which point the $a g r$ quorum-sensing system is activated (10). This interbacterial chemical communication causes the upregulation of RNAIII, the main effector molecule of the agr system, which results in the production of a variety of virulence factors and subsequent tissue necrosis (11). Wound infection is defined as the presence of replicating organisms within a wound with subsequent host injury (6). The transition from the bacterial colonization form to the sessile biofilm form makes the infection more complex, further damaging wound healing and reepithelialization $(12,13)$.

In recent years, there has been increasing interest in the use of negative pressure wound therapy (NPWT) for the treatment of infected wounds due to its efficacy (14-16). Despite growing research concerning the low incidence of biofilm-associated infections, the mechanism of NPWT in infection control remains to be elucidated, particularly with regard to the changes of bacteria secondary to NPWT $(17,18)$. $S$. aureus responds to physical stimulations by regulating gene expression (19-22). Therefore, further studies are urgently required to determine the existent form and gene expression pattern of $S$. aureus in the microenvironment established by NPWT.

The aim of the present study was to investigate and evaluate the response of $S$. aureus to NPWT during acute wound infection. An established rabbit model was used to examine the distribution and existing form of S.aureus in tissues. Bacterial burden was also determined to confirm the survival and persistence of bacteria. The adaptive expression of bacterial genes associated with colonization and biofilm regulation secondary to NPWT was further monitored. These experiments aimed to develop an improved understanding of the role of NPWT in bacterial existent form and infection control, setting a foundation for further elucidation of its mechanism.

\section{Materials and methods}

Animal ethics. A total of 18 1-year-old female Japanese large-ear white rabbits $(\sim 3 \mathrm{~kg}$, purchased from the Laboratory Animal Centre of the Academy of Military Medical Sciences) were used for the present study. The animals were acclimated to standard housing and fed ad libitum under a constant temperature $\left(22^{\circ} \mathrm{C}\right)$ and humidity $(45 \%)$ with a $12 \mathrm{~h} \mathrm{light} / \mathrm{dark}$ cycle. All experiments were approved by the Medical Ethics Committee of the Chinese People's Liberation Army (PLA) General Hospital (approval no. 2014-X9-8) in compliance with the Guidelines for Care and Use of Animals in Research (23).
Bacterial strain and culture. S. aureus strain RN6390 with constitutive green fluorescent protein (GFP) expression (obtained from the Chinese PLA Institute for Disease Control and Prevention) was cultured in Luria-Bertani (LB) broth in a rotary shaker at $200 \mathrm{rpm}$ and $37^{\circ} \mathrm{C}$. S. aureus was grown overnight and subcultured until the log phase. To prepare the inoculum, bacteria were collected by centrifugation $(5,000 \mathrm{x} \mathrm{g}$, $4^{\circ} \mathrm{C}, 10 \mathrm{~min}$ ), washed thrice with PBS and suspended to an optical density value of 1.0 at $600 \mathrm{~nm}$ measured using an ultraviolet spectrophotometer (GeneQuant 1300; Biochrom, Ltd.) in PBS, equivalent to $1 \times 10^{5}$ colony-forming units (CFUs) $/ \mu 1$ empirically (24).

Rabbit model of acute wound infection. The wounding and bacterial inoculation protocol was based on a previously published wound model with minor modifications $(25,26)$. In brief, rabbits were anesthetized by intramuscular injection of ketamine $(45 \mathrm{mg} / \mathrm{kg})$ and xylazine $(5 \mathrm{mg} / \mathrm{kg})$ mixture. The back of each animal was shaved and residual hair was removed with depilatory cream. The surgical site was sterilized twice with $70 \%$ ethanol. A standardized $3 \mathrm{~cm}$-diameter full-thickness dermal wound was created below the dorsal muscle on each side of the back. Following hemostasis and confirmation of no active bleeding, each wound was inoculated with a total of $1 \times 10^{7} \mathrm{CFUs}$ of $S$. aureus at a volume of $0.1 \mathrm{ml}$. The wounds were bandaged with sterile gauze dressings. Bacteria were allowed to proliferate in vivo for a minimum of $6 \mathrm{~h}$ to ensure bacterial adhesion and colonization (27). All procedures in the present study were performed by the same surgeon.

Study design and treatment protocol. All rabbits were used to create acute wound infection models (two wounds/rabbit). For each animal, the two wounds were respectively and randomly assigned to the 'untreated control side' and the 'NPWT side'. The rabbits were divided into two groups: Group A (bacterial count, gene expression analysis and healing condition, $\mathrm{n}=10$ ) and group B (histological study, $n=8$ ).

The wounds were treated based on a previously published protocol with minor modifications $(25,26)$. In brief, at $6 \mathrm{~h}$ post inoculation, the wound on the 'NPWT side' of each rabbit was dressed with the standard NPWT dressing (consisting of polyvinyl alcohol foam, semiocclusive transparent dressing and suction tube; Wuhan VSD Medical Science and Technology Co., Ltd.) trimmed to the appropriate size in advance. The suction tube was then connected to the vacuum pump device (kindly provided by Professor Lei Hu, Beihang University, Beijing, China). The rabbits were single-caged and the vacuum pump device was placed outside the cage. The tubing and the wound area were swathed in bandages to prevent the rabbits from biting it. Wounds treated with NPWT were subjected to continuous negative pressure at $-125 \mathrm{mmHg}$. Dressings were checked daily and changed every $48 \mathrm{~h}$ at most, as recommended for infected wounds by the manufacturer. If the wound required imaging on that day, dressings were also changed. After a period of 8 days of treatment, the rabbits in group B were euthanized. The healing conditions of the rabbits in group A were observed until the 28th day after operation. The wound on the contralateral side of each rabbit was bandaged and followed the same protocol as the treated wound, acting as 
the untreated control. All animals in group A were euthanized at the end of the present study.

Histological analysis. Samples $(1 \times 1 \times 1 \mathrm{~cm})$ from the center of the wounds were harvested immediately after animal euthanasia on postoperative day 8 . Each sample was equally divided into three parts, which were used for laser scanning confocal microscopy (LSCM), hematoxylin and eosin (H\&E) staining and scanning electron microscopy (SEM).

To visualize the distribution of GFP-labeled S. aureus in the tissue, the specimens were embedded in optimal cutting temperature compound (Sakura Finetek USA, Inc.), subsequently snap-frozen and stored in liquid nitrogen until cryosectioning. Cryosections measuring $6 \mu \mathrm{m}$ at the vertical section were obtained using a Leica CM1950 freezing microtome (Leica Microsystems $\mathrm{GmbH}$ ). Observation of section slides was performed using an FV1000 confocal laser scanning microscope (Olympus Corporation; x200 magnification). For better evaluation, transmitted light images were captured and merged with GFP-fluorescent images (5).

For histopathological analysis, duplicate samples from the wounds above were fixed in $10 \%$ neutral formalin for $24 \mathrm{~h}$ at $25^{\circ} \mathrm{C}$, embedded in paraffin, sectioned vertically into $4 \mu \mathrm{m}$ slices and stained with H\&E. Images were captured with an Olympus BX51 microscope (Olympus Corporation) equipped with an Olympus DP71 digital camera (Olympus Corporation).

Duplicate samples from the wounds above were fixed in $2.5 \%$ glutaraldehyde $(24 \mathrm{~h})$ and $1 \%$ osmium tetroxide $(12 \mathrm{~h})$ at $25^{\circ} \mathrm{C}$, dehydrated through an ethanol series and hexamethyldisilazane, mounted to specimen stubs using double-sided tape and coated with gold in an auto sputter coater (TedPella, Inc.). Imaging of the tissue samples was performed using a scanning electron microscope (S-3400N; Hitachi, Ltd.). Digital images were captured using the same parameters $(15.0 \mathrm{kV}, x 5,000$ magnification) and analyzed by three blinded experienced observers (one microbiologist and two pathologists). Wound sample and granulation tissue was evaluated as described by Kamamoto et al (28). Bacterial aggregates and necrosis were observed in the region of interest, which were regions with bacterial aggregates and necrosis below the wound surface. Five sections were evaluated per sample.

Viable bacteria count measurement. Wound samples were harvested under anesthesia as described by Morykwas et al (29) on postoperative days 1, 4 and 8 . In brief, each wound was equally divided into four quadrants. Three samples $(\sim 0.1 \mathrm{~g}$ in total) were randomly obtained with biopsy forceps in one of the quadrants at each time point (days 1,4 and 8). Specimens were immediately weighed and homogenized into a $1 \mathrm{ml}$ suspension with sterile PBS at $4^{\circ} \mathrm{C}$. The homogenates were then serially diluted, plated on Staphylococcus Isolation Agar (Hardy Diagnostics) and incubated at $37^{\circ} \mathrm{C}$ for $24 \mathrm{~h}$. The standard colony-counting method (25) was performed and the results were expressed as the logarithm of CFUs per $g$ of tissue.

$R N A$ isolation and reverse transcription-quantitative PCR (RT-qPCR). Wound samples ( 0.1g for both groups) collected under anesthesia on postoperative days 1,2, 4 and 8 were snap-frozen and stored in liquid nitrogen until RNA extraction and subsequent RT-qPCR analysis as previously described (5). Briefly, frozen tissue was pulverized using a hammer, homogenized in the presence of cell disruption solution (cat. no. 4305895; Thermo Fisher Scientific, Inc.) and lysozyme (AC142; Omega Bio-Tek, Inc.) and incubated at $37^{\circ} \mathrm{C}$ for $10 \mathrm{~min}$. Total RNA was extracted using a RNAprep Pure Cell/Bacteria kit (Tiangen Biotech Co., Ltd.) according to the manufacturer's instructions. Isolated RNA was treated with Recombinant DNase I (Takara Bio, Inc.) at $37^{\circ} \mathrm{C}$ for 20 min to remove contaminating DNA. RNA quality and concentration were assessed using a Qubit ${ }^{\circledR} 2.0$ Fluorometer (Thermo Fisher Scientific, Inc.). RNA was reverse transcribed into cDNA using a TIANScript RT kit (Tiangen Biotech Co., Ltd.) according to the manufacturer's instructions. The primer pairs used in the present study are listed in Table I. Transcript levels of the selected $S$. aureus genes were evaluated by RT-qPCR analysis using a KAPA SYBR Fast qPCR kit (Roche Diagnostics GmbH) on a 7900HT Fast Real-Time PCR system (Applied Biosystems; Thermo Fisher Scientific, Inc.). The following thermocycling conditions were used for the PCR: Initial denaturation at $95^{\circ} \mathrm{C}$ for $3 \mathrm{~min} ; 40$ cycles of $95^{\circ} \mathrm{C}$ for $3 \mathrm{sec}, 60^{\circ} \mathrm{C}$ for $20 \mathrm{sec}$; and one dissociation step at $95^{\circ} \mathrm{C}$ for $15 \mathrm{sec}, 60^{\circ} \mathrm{C}$ for $15 \mathrm{sec}$ and $95^{\circ} \mathrm{C}$ for $15 \mathrm{sec}$. All samples were analyzed in triplicate and $\mathrm{Ct}$ values were normalized against $16 \mathrm{~S}$ ribosomal RNA expression. The $2^{-\Delta \Delta \mathrm{Cq}}$ method (30) was used to determine the relative expression levels of $S$. aureus genes in accordance with the manufacturer's protocol. A total of $200 \mu \mathrm{l}$ of the inoculum was retained when establishing the animal model and snap-frozen in liquid nitrogen. The inoculum was used as the calibrator with a value of 1 . The results were expressed as the mean fold-change of gene expression level relative to that of the same gene found in the inoculum.

Measurement of wound closure. Images of the wounds in group A were captured with a digital camera (IXUSi; Canon, Inc.) every 3 days until postoperative day 28 . All images were independently analyzed by two expert pathologists to evaluate and delimit the epithelialisation boundary. The wound area was then calculated using ImagePro Plus version 6.0 software (Media Cybernetics, Inc.) with the scale set using the ruler in the image. The rate of wound closure was expressed as the percentage of the initial wound area.

Statistical analysis. Experimental data were analyzed using a mixed ANOVA followed by Bonferroni's post hoc test to evaluate the difference between groups at each time point. All analyses were performed using SPSS 17.0 software (SPSS, Inc.) and data were presented as the mean \pm standard deviation. $\mathrm{P}<0.05$ was considered to indicate a statistically significant difference.

\section{Results}

Histological examination and detection of S. aureus in soft tissue. Sections of samples from the affected wounds at the untreated control side presented with numerous discrete aggregates and microcolonies of bacteria (indicated by black arrows; $\sim 100 \mu \mathrm{m}$ in size; Fig. 1A) located in the tissue, observed by conventional light microscopy on postoperative day 8 . These bacterial colonies caused local necrosis, leaving cavities with distinct boundaries (indicated by black arrows). However, 


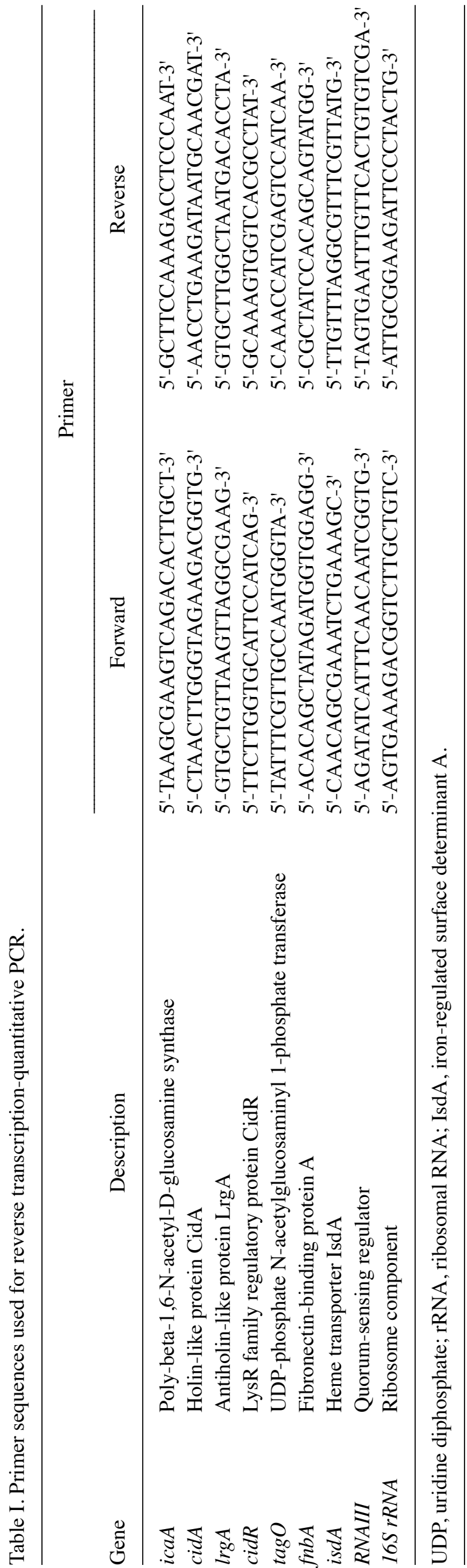

bacterial aggregates were not detected at the NPWT side, instead presenting with a healthier granulation bed without local necrosis (Fig. 1B). LSCM utilizing GFP-tagged $S$. aureus showed that bacteria appeared as clusters and biofilms $(\sim 100 \mu \mathrm{m}$ in size) in untreated wounds, resulting in distinct regions in deep tissue layers (indicated by white arrows; $\sim 300 \mu \mathrm{m}$ in depth; Fig. 1C). In comparison, bacteria in wounds treated with NPWT were sparsely visible and dispersedly distributed in the tissue (Fig. 1D). To confirm these results, SEM was performed, presenting with numerous $S$. aureus microcolonies (indicated by white arrows) embedded within the lattice-like extracellular matrix in untreated wounds (Fig. 1E). Conversely, the bacteria in tissues treated by NPWT appeared as single cells or diplococci, lacking an extracellular matrix (Fig. 1F).

Viable bacteria count measurement. To investigate the effects of NPWT on the survival of $S$. aureus, bacterial burden was determined at multiple time points (Fig. 2). On postoperative day 1 , there was no statistical difference between untreated and NPWT groups, with an average of $4.05 \times 10^{7} \mathrm{CFUs}$ per $\mathrm{g}$ of tissue. Bacterial counts of untreated wounds showed a consistent level of viable bacteria throughout the course of time. In contrast, there was a significant decrease in viable bacteria levels in the NPWT group compared with the untreated group, verifying the consistency and reproducibility of the results in our previous work (25). Nevertheless, NPWT only led to a reduction in bacterial counts by one-log fold on day 8 , with an average of $1.16 \times 10^{6} \mathrm{CFUs} / \mathrm{g}$, which was still a considerable amount of bacteria in the tissue.

Effect of NPWT on the transcription of S. aureus biofilm regulators. To investigate the mechanism of NPWT in affecting the aggregation of $S$. aureus in tissue, the transcription of biofilm regulators during the process of wound infection was analyzed using RT-qPCR. The relative expression ratio of each gene was expressed as the fold-change relative to the level found in the inoculum. In the control group, a 2.6-fold increase for poly-beta-1,6-N-acetyl-D-glucosamine synthase (icaA) (Fig. 3A) was observed on post operative day 1 , after which the expression further increased and reached a maximum of 6.8-fold on day 4. Expression then fell to 5.3-fold on day 8. Similar to icaA, the transcription of holin-like protein CidA (cidA) (Fig. 3B) gradually increased and reached a maximal 2.5 -fold increase on day 4 , then declined to 2.0 -fold on day 8 . The expression of antiholin-like protein $\operatorname{LrgA}(\operatorname{lrg} A)$ (Fig. 3C) increased and remained 4-fold higher compared with baseline levels over time. After an initial decline on day 1 and 2, the expression of cidR (Fig. 3D) returned to baseline levels at day 4 and 8 . In comparison, NPWT resulted in a significant decrease in the expression of icaA and $\operatorname{cid} A$ and a significant increase in $\operatorname{lrg} A$ levels compared with that in the control group at days 1,2, 4 and 8 , but did not have any significant effect on cidR expression (Fig. 3).

Changes in $S$. aureus colonization under NPWT treatment. To further understand the living state of S. aureus in tissue, the adaptive expression of bacterial genes associated with colonization during wound infection was investigated. In the control group, the expression of tagO (Fig. 4A) continued to decrease in a time-dependent manner and reached $17 \%$ of inoculum levels on day 8 . Conversely, a 14-fold increase of 
A

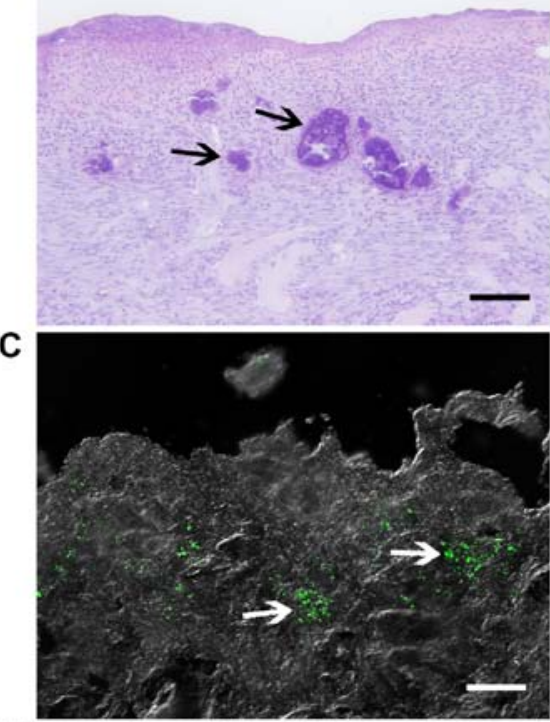

E

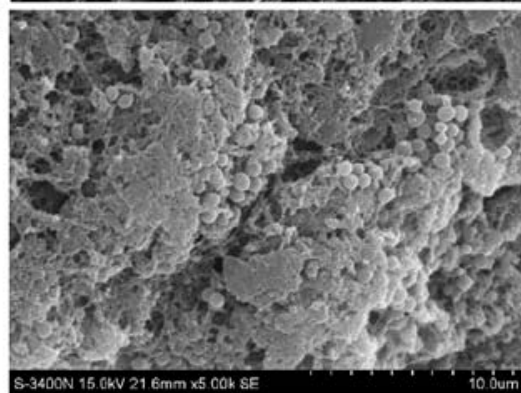

B

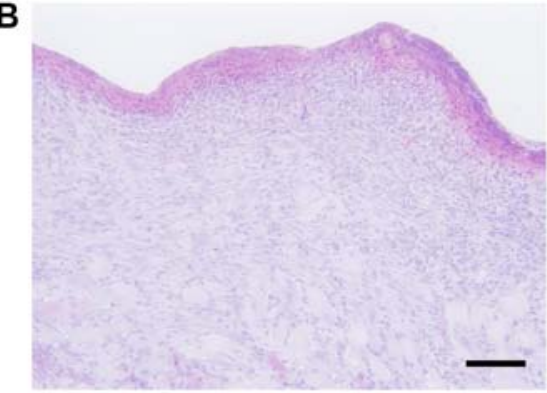

D

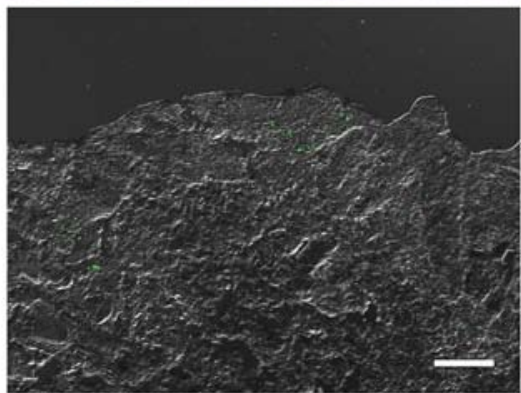

$\mathbf{F}$

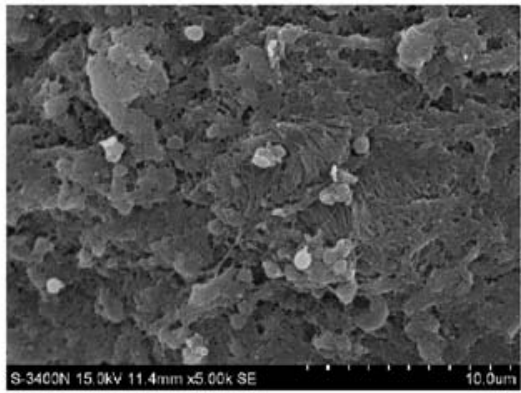

Figure 1. Histological examination and detection of Staphylococcus aureus in soft tissue. (A) Hematoxylin and eosin staining overview of wound tissue at the vertical section presented with numerous discrete bacterial aggregates and local necrosis (indicated by black arrows) in untreated wounds on day 8 . (B) Bacterial aggregates and necrosis area were not detected in the NPWT group. (C) Laser scanning confocal microscopy showed that green fluorescent protein-tagged S. aureus (green) appeared as microcolonies (indicated by white arrows) in untreated wounds. (D) S. aureus in wounds treated with NPWT were dispersedly distributed in the tissue as single cells or diplococci, without bacterial colonies. (E) Scanning electron microscopy presented with numerous microcolonies of $S$. aureus (indicated by white arrows) embedded within the latticelike extracellular matrix in untreated wounds. (F) The bacteria in tissues treated by NPWT appeared as single cells or diplococci, lacking in extracellular matrix. Scale bar=100 $\mu \mathrm{m}$. NPWT, negative pressure wound therapy.

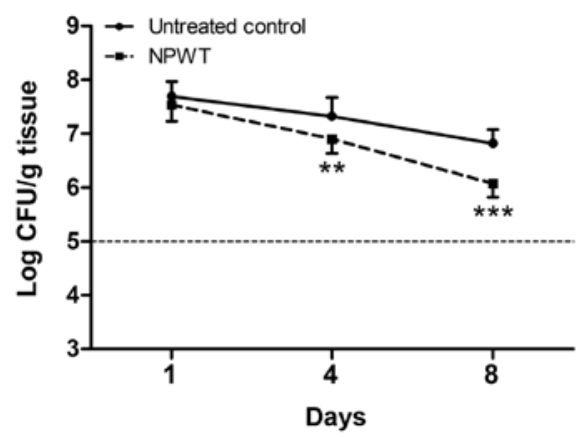

Figure 2. Viable bacteria count measurements. Bacterial counts of untreated wounds showed a consistent level throughout the time course, averaging about $1 \times 10^{7} \mathrm{CFUs} / \mathrm{g}$ tissue. By contrast, there was a moderate decrease in the bacterial counts in the NPWT group, with approximately $1 \times 10^{6} \mathrm{CFUs} / \mathrm{g}$ tissue on day $8 .{ }^{* *} \mathrm{P}<0.01$ and ${ }^{* * * *} \mathrm{P}<0.001$ vs. untreated control. $\mathrm{N}=10$ wounds group. CFU, colony-forming unit; NPWT, negative pressure wound therapy.

fnbA levels (Fig. 4B) was observed compared with inoculum levels on day 1 , after which the expression gradually increased and reached 52-fold of baseline levels on day 8 . The expression of isd A (Fig. 4C) followed a similar pattern, with a 20 -fold increase at day 1 and a gradual increase to 34-fold at day 8 . RNAIII expression (Fig. 4D) increased more slowly compared with fnbA and isdA levels and reached a maximum of 3.5-fold increased compared with baseline levels on day 2. Expression started to decrease at days 4 and 8 , but remained elevated with a 2.2-fold increase above baseline levels on day 8. In contrast, NPWT led to significant upregulation of $\operatorname{tag} \mathrm{O}$, fnbA and isdA levels, and significant downregulation of RNAIII levels compared with the controls at days 1, 2, 4 and 8 (Fig. 4).

Wound healing condition. After 8 days of treatment, the wound healing conditions of both groups were observed until postoperative day 28. Gross appearance on the 18th day manifested with a small wound area with clean granulation tissue bed and without necrosis or purulent secretion in the NPWT group (Fig. 5A) compared with the control (Fig. 5B). On the 24th day, the wound treated with NPWT completely healed (Fig. 5C), with a neoepithelium covering the wound center. By contrast, a large skin defect presented in the control group (Fig. 5D), with film-like exudates overlying the wound bed. Although treatment was only administered for 8 days, wound healing of the NPWT group was significantly improved compared with the control group throughout time period (Fig. 5E). 

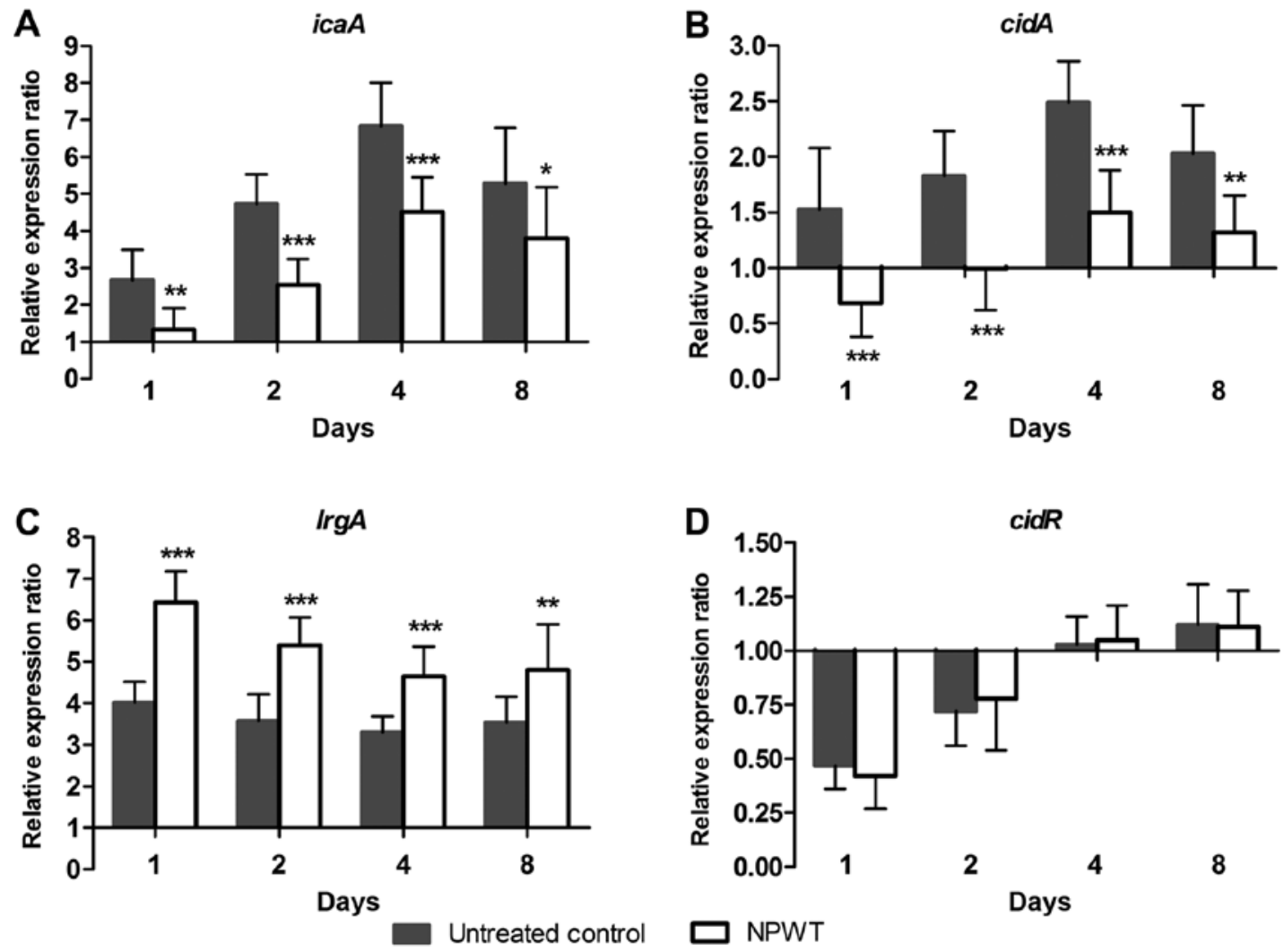

Figure 3. Reverse transcription-quantitative PCR analysis of Staphylococcus aureus biofilm regulators in response to NPWT. Transcript levels of S. aureus biofilm regulators for both groups are presented as fold-changes relative to the inoculum, which was used as the calibrator with a value of 1. (A-C) NPWT led to a significant decrease in the transcription of $i c a A$ and $c i d A$ and an increase in $\operatorname{lrg} A$ compared with untreated wounds throughout the time course, (D) but did not show any significant affect on $c i d R$ levels. ${ }^{*} \mathrm{P}<0.05,{ }^{* * *} \mathrm{P}<0.01$ and ${ }^{* * * *} \mathrm{P}<0.001$ vs. untreated control. $\mathrm{n}=10$ wounds/group. NPWT, negative pressure wound therapy; icaA, poly-beta-1,6-N-acetyl-D-glucosamine synthase; $c i d A$, holin-like protein CidA; $\operatorname{lrg} A$, antiholin-like protein LrgA; $c i d R$, LysR family regulatory protein CidR.
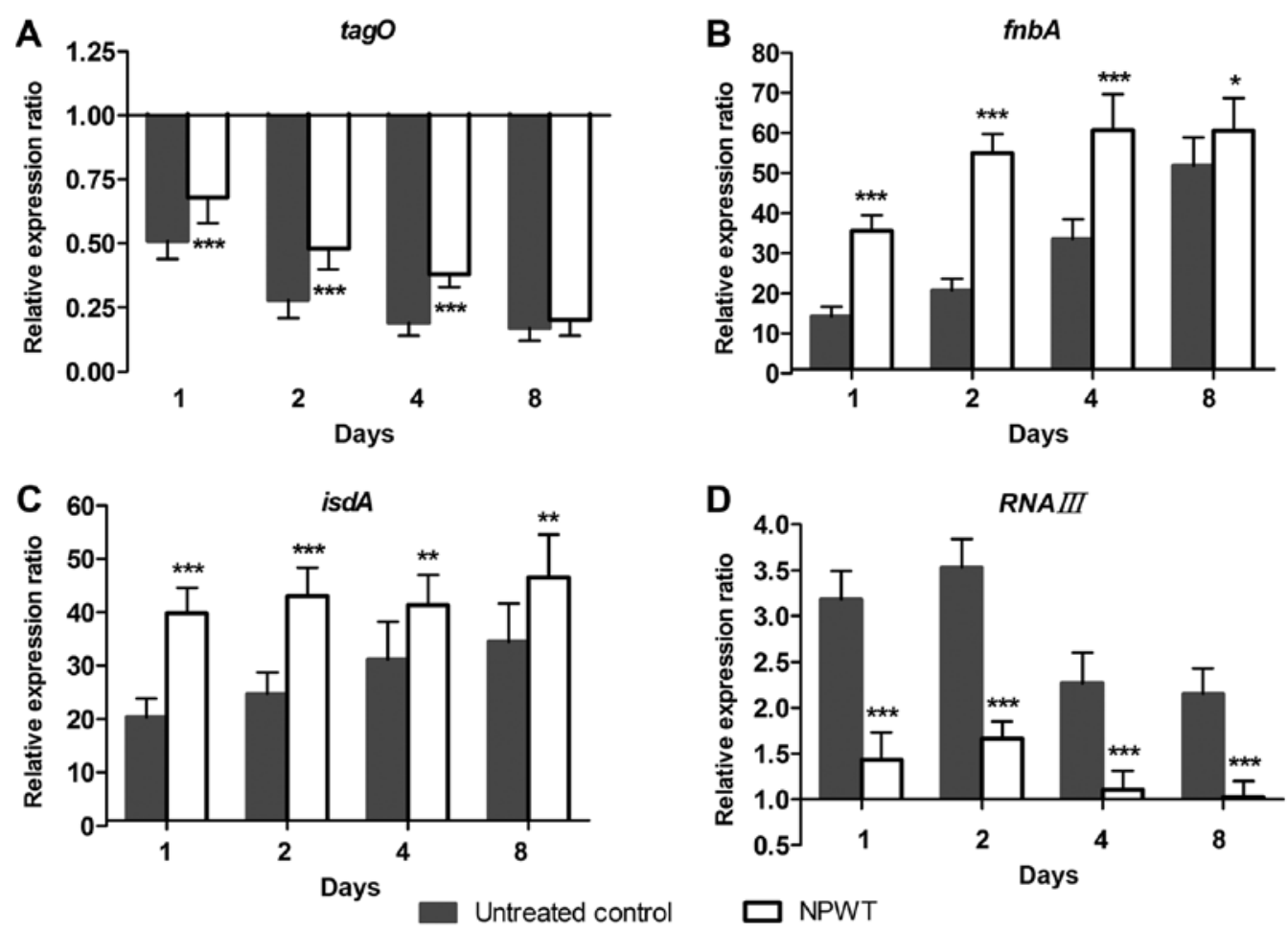

Figure 4. Changes in the transcription of genes involved in Staphylococcus aureus colonization in response to NPWT. (A-C) NPWT resulted in significant upregulation of $\operatorname{tag} O, f n b A$ and $i s d A$ levels compared with untreated wounds throughout the time course. (D) RNAIII levels in the NPWT group were downregulated relative to the control group. Data of both groups were presented as the fold-changes in gene expression relative to the inoculum, which was used as the calibrator with a value of $1 .{ }^{*} \mathrm{P}<0.05,{ }^{* * *} \mathrm{P}<0.01$ and ${ }^{* * * *} \mathrm{P}<0.001$ vs. untreated control. $\mathrm{n}=10$ wounds/group. NPWT, negative pressure wound therapy. tagO UDP-phosphate $\mathrm{N}$-acetylglucosaminyl 1-phosphate transferase; $f n b A$, fibronectin-binding protein $\mathrm{A}$; isd $\mathrm{A}$, iron-regulated surface determinant $\mathrm{A}$. 

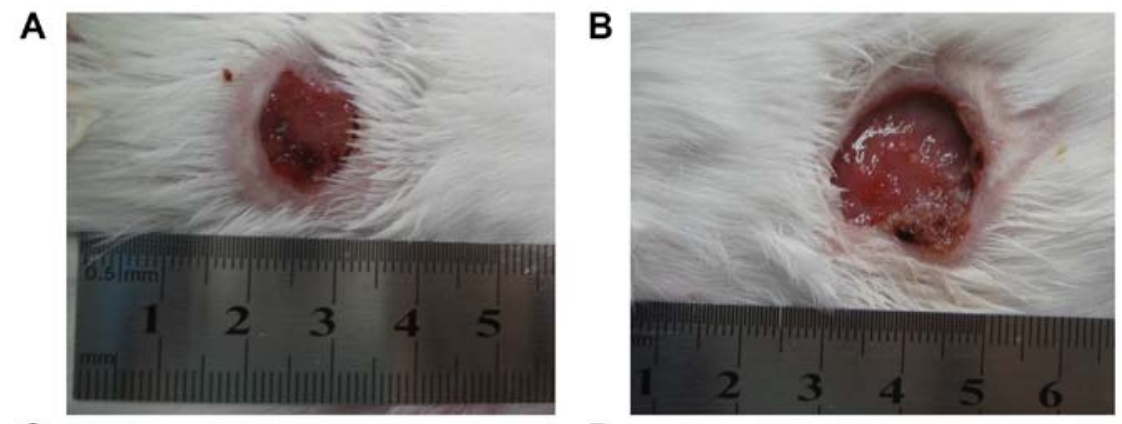

C

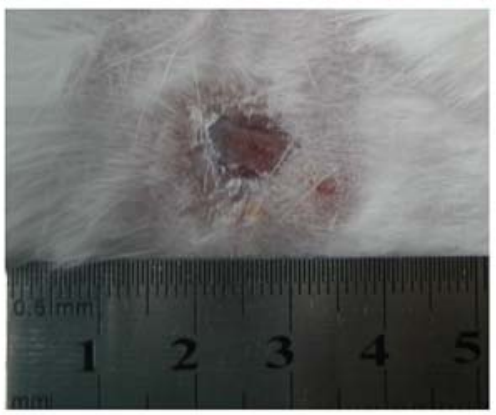

E
D

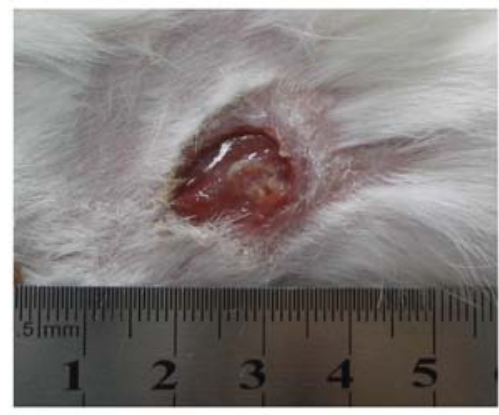

$\rightarrow$ Untreated control

$\rightarrow$ NPWT

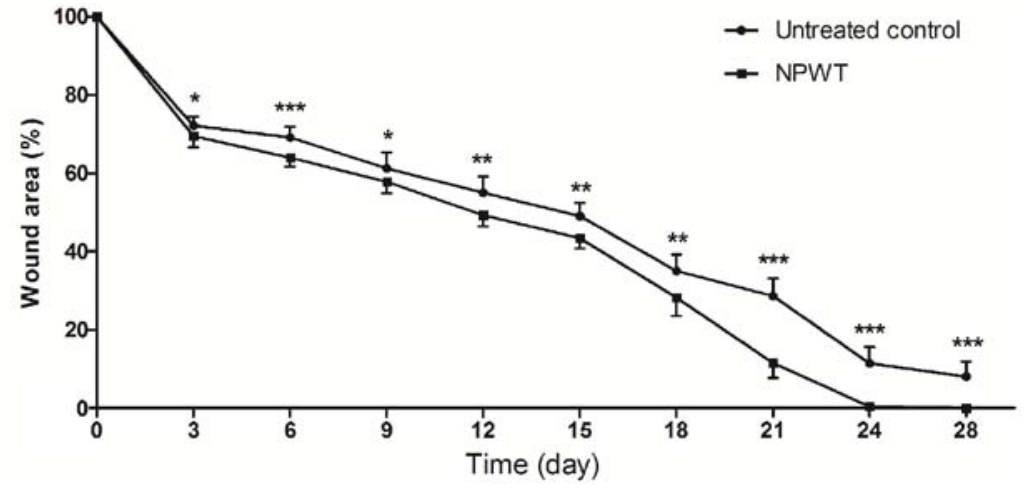

Figure 5. Gross appearance of the wounds. (A) On the 18th day, the wounds treated by NPWT manifested a small and clean wound bed, compared with (B) untreated wounds. (C) The wounds treated by NPWT healed completely on the 24th day. (D) Conversely, there was a delay in healing and film-like exudates covering the untreated wounds. (E) The healing condition of the NPWT group was significantly better compared with the untreated wounds throughout the time course. Data are presented as the percentage of initial wound area. ${ }^{*} \mathrm{P}<0.05,{ }^{* *} \mathrm{P}<0.01$ and ${ }^{* * *} \mathrm{P}<0.001$ vs. untreated control. $\mathrm{n}=10$ wounds/group. NPWT, negative pressure wound therapy.

\section{Discussion}

The pathogenesis of wound infection has long been considered to be complex and multifactorial (31). Bacterial amount and invasiveness, as well as the immune competencies of the host, affect the outcome of wound infection $(25,26)$. As the main cause behind wound healing impairment, bacterial biofilm and quorum-sensing make the treatment of infected wounds much more challenging (31-33). With almost 1,000-fold higher antimicrobial resistance compared with planktonic bacteria, biofilms develop rapidly and present either at the surface or within the profound layers of wounds (34). In an effort to identify novel approaches to wound care and infection control, NPWT was demonstrated to be safe and effective in avoiding biofilm-associated infections as a physical therapy $(14,16,35)$. However, the mechanism involved remains to be elucidated, particularly with regard to the response of bacteria secondary to NPWT. It is well known that bacterial gene expression is highly affected by the microenvironment (21). Transcriptomic research demonstrated that quorum sensing is downregulated in infections in vivo compared with in vitro conditions, suggesting bacterial physiology differs in clinical infections relative to in vitro experiments (36). The present study investigated the distribution and existing form of $S$. aureus in the local microenvironment established by NPWT and monitored the adaptive expression of bacterial genes during acute wound infection in vivo.

The results of the present study indicated that NPWT had an obvious influence on the aggregation and distribution of S. aureus in the tissue. In the present study, bacterial aggregates were identified in the control group by histological evaluation, along with distinct regions and micronecrosis under the wound surface. This verified the persistence of bacteria within the tissue for at least 8 days, mainly in the form of microcolonies with extracellular matrix. However, NPWT led to a scattering distribution of bacteria in wounds, accompanied by a healthier granulation bed. Although the dispersive distribution of bacteria is associated with active infection in some cases, wound healing was improved by NPWT in the present study, which was also proven by extensive clinical research $(14,15,37)$. 
To investigate the effect of NPWT on the survival of $S$. aureus in wounds and the relationship between the dispersive distribution and bacterial burden, viable bacteria counts were measured. The present data showed a decrease in viable bacteria in the NPWT group compared with the untreated control. Nevertheless, the bacterial load was not dramatically reduced in comparison with the control even at day 8 , with $\sim 1 \times 10^{6} \mathrm{CFUs} / \mathrm{g}$, which was still a considerable amount of bacteria in the tissue. This indicated that NPWT did not directly kill bacteria in wounds and the dispersive distribution of $S$. aureus should not be attributed to a low bioburden (38).

$S$. aureus responded to physical stimulations by altering gene transcription (19-22). To better understand the effects of NPWT on the living state of $S$. aureus in wounds, the adaptive expression of bacterial biofilm regulators during wound infection was studied. Transcriptional analysis showed that there were significant inhibitions on the expression of genes involved in the production of biofilm components, including ica $A$ and $\operatorname{cid} A$. Furthermore, the transcription of $\operatorname{lrg} A$, which encodes an antiholin-like protein with an inhibitory effect on murein hydrolase activity (9), was increased in the NPWT group. These results suggested that NPWT inhibited the biosynthesis of the biofilm matrix, leading to a free-living growth pattern for bacteria in the tissue. To some extent, this may explain the dispersive distribution of $S$. aureus in the present study and the low incidence of biofilm-associated infections in the clinical use of NPWT.

To further explore the events involved in the shift of phenotypic growth pattern, the expression of adhesion factors associated with bacterial colonization was analyzed. The present data showed that NPWT led to increased expression of the adhesion factors, suggesting an intensive colonization of $S$. aureus in tissues. The colonization state alone was reported to do little harm to host cells and does not delay the wound healing process $(6,7)$. The life styles of $S$. aureus in colonization or spreading invasive infection were demonstrated to be regulated by the agr quorum-sensing system, a cell density-dependent regulatory mechanism (11). To determine whether the discrepancy between bacterial dispersive distribution and enhanced colonization in the NPWT group could be ascribed to the low activity of quorum sensing, the transcript analysis of RNAIII, the main effector molecule of the agr system, was included in the present study. The results showed that RNAIII transcript levels were significantly reduced in the NPWT group compared with the untreated control group, suggesting a decreased activity of the agr system. A possible explanation for this decrease may be that the bacterial density in NPWT-treated wounds was under the threshold for agr activation, thus not leading to active and invasive infection $(11,32)$. Bacterial dispersive distribution and enhanced colonization appears to be a more uniform scenario, which reflects the commensalism of S. aureus in the local niche built by NPWT (39). This suggested that bacteria benefitted from the host but does little harm to the wound repair and healing process.

However, the present study exhibited a number of limitations. Although the data of bacterial gene expression lend support to the distribution and existing form of S. aureus in tissues, the exact mechanism involved remains to be elucidated. As a physical therapy based on continuous suction, NPWT might affect the pathogenesis of S.aureus infection by multiple ways and means, including stereotaxic drainage, pressure variation and shear stress $(40,41)$. In particular, multiple sampling and further analysis on wound healing was performed to reduce the error caused by individual differences. Biopsies were carefully performed to ensure that other analyses are not affected. Despite concerns about healing and disruption of the bacterial state by repeated sampling, the actual impact was marginal (42). Only the transcription of genes related to biofilm formation and bacterial colonization was investigated in the present study. As the process of infection involves numerous gene regulatory networks, it is necessary to study global gene expression to validate the present findings in future work.

In summary, the present findings indicated that $S$. aureus responds to NPWT by regulating gene expression, manifesting a decrease in biofilm formation and an increase in bacterial adhesion. The transition from bacterial biofilm to less invasive colonization may reflect a commensal relationship between $S$. aureus and host cells, which potentially benefits the wound repair and healing process. Further studies into the processes influencing the divergence between biofilm infection and harmless colonization may aid in the discovery of novel therapeutics against infections in the field of wound care.

\section{Acknowledgements}

Not applicable.

\section{Funding}

The present study was supported by the National Natural Science Foundation of China (grant no. 81472112).

\section{Availability of data and materials}

The datasets used and/or analyzed during the present study are available from the corresponding author on reasonable request.

\section{Authors' contributions}

TL, GW, LZ and PT performed the animal studies. GW, PY and ZL performed histological experiments. TL, PY and ZL performed bacterial counting and RT-qPCR experiments. TL, GW, PY and PT analyzed and interpreted the data. TL and PT prepared the manuscript. All authors read and approved the final manuscript.

\section{Ethics approval and consent to participate}

All experiments were approved by the Medical Ethics Committee of the Chinese PLA General Hospital (approval no. 2014-X9-8) in compliance with the Guidelines for Care and Use of Animals in Research (8th edition).

\section{Patient consent for publication}

Not applicable.

\section{Competing interests}

The authors declare that they have no competing interests. 


\section{References}

1. Marimuthu K, Eisenring MC, Harbarth S and Troillet N Epidemiology of Staphylococcus aureus surgical site infections. Surg Infect (Larchmt) 17: 229-235, 2016.

2. Heilmann C: Adhesion mechanisms of staphylococci. Adv Exp Med Biol 715: 105-123, 2011.

3. Speziale P, Pietrocola G, Rindi S, Provenzano M, Provenza G Di Poto A, Visai L and Arciola CR: Structural and functional role of Staphylococcus aureus surface components recognizing adhesive matrix molecules of the host. Future Microbiol 4 : $1337-1352,2009$.

4. Weidenmaier C, Kokai-Kun JF, Kulauzovic E, Kohler T, Thumm G, Stoll H, Götz F and Peschel A: Differential roles of sortase-anchored surface proteins and wall teichoic acid in Staphylococcus aureus nasal colonization. Int J Med Microbiol 298: 505-513, 2008

5. Burian M, Rautenberg M, Kohler T, Fritz M, Krismer B, Unger C Hoffmann WH, Peschel A, Wolz C and Goerke C: Temporal expression of adhesion factors and activity of global regulators during establishment of Staphylococcus aureus nasal colonization. J Infect Dis 201: 1414-1421, 2010.

6. Edwards R and Harding KG: Bacteria and wound healing. Curr Opin Infectious Dis 17: 91-96, 2004.

7. Poutahidis T, Kearney SM, Levkovich T, Qi P, Varian BJ, Lakritz JR, Ibrahim YM, Chatzigiagkos A, Alm EJ and Erdman SE: Microbial symbionts accelerate wound healing via the neuropeptide hormone oxytocin. PLoS One 8: e78898, 2013.

8. Lee K, Lee JH, Ryu SY, Cho MH and Lee J: Stilbenes reduce Staphylococcus aureus hemolysis, biofilm formation, and virulence. Foodborne Pathog Dis 11: 710-717, 2014.

9. Bayles KW: The biological role of death and lysis in biofilm development. Nat Rev Microbiol 5: 721-726, 2007.

10. Otto M: Staphylococcal infections: Mechanisms of biofilm maturation and detachment as critical determinants of pathogenicity. Annu Rev Med 64: 175-188, 2013.

11. Thoendel M, Kavanaugh JS, Flack CE and Horswill AR: Peptide signaling in the staphylococci. Chem Rev 111: 117-151, 2011.

12. Schierle CF, De la Garza M, Mustoe TA and Galiano RD: Staphylococcal biofilms impair wound healing by delaying reepithelialization in a murine cutaneous wound model. Wound Repair Regen 17: 354-359, 2009.

13. Roche ED, Renick PJ, Tetens SP, Ramsay SJ, Daniels EQ and Carson DL: Increasing the presence of biofilm and healing delay in a porcine model of MRSA-infected wounds. Wound Repair Regen 20: 537-543, 2012.

14. Anagnostakos K and Mosser P: Negative pressure wound therapy in the management of postoperative infections after musculoskeletal tumour surgery. J Wound Care 23: 191-194, 196-197, 2014.

15. Hahn HM, Lee IJ, Woo KJ and Park BY: Silver-impregnated negative-pressure wound therapy for the treatment of lower-extremity open wounds: A prospective randomized clinical study. Adv Skin Wound Care 32: 370-377, 2019.

16. Singh DP, Gowda AU, Chopra K, Tholen M, Chang S, Mavrophilipos V, Semsarzadeh N, Rasko Y and Holton Iii L: The effect of negative pressure wound therapy with antiseptic instillation on biofilm formation in a porcine model of infected spinal instrumentation. Wounds 28: 175-180, 2017.

17. Glass GE, Murphy GRF and Nanchahal J: Does negative-pressure wound therapy influence subjacent bacterial growth? A systematic review. J Plast Reconstr Aesthet Surg 70: 1028-1037, 2017.

18. Ćirković I, Jocić D, Božić DD, Djukić S, Konstantinović N and Radak D: The effect of vacuum-assisted closure therapy on methicillin-resistant Staphylococcus aureus wound biofilms Adv Skin Wound Care 31: 361-364, 2018.

19. Islam N, Kim Y, Ross JM and Marten MR: Proteomic analysis of Staphylococcus aureus biofilm cells grown under physiologically relevant fluid shear stress conditions. Proteome Sci 12: 21, 2014.

20. Castro SL, Nelman-Gonzalez M, Nickerson CA and Ott CM: Induction of attachment-independent biofilm formation and repression of Hfq expression by low-fluid-shear culture of Staphylococcus aureus. Appl Environ Microbiol 77: 6368-6378, 2011.

21. Rosado H, Doyle M, Hinds J and Taylor PW: Low-shear modelled microgravity alters expression of virulence determinants of Staphylococcus aureus. Acta Astronautica 66: 408-413, 2010.

22. Li T, Wang G, Yin P, Li Z, Zhang L, Liu J, Li M, Zhang L, Han L and Tang P: Effect of negative pressure on growth, secretion and biofilm formation of Staphylococcus aureus. Antonie van Leeuwenhoek 108: 907-917, 2015.
23. Institute of Laboratory Animal Resources (US). Committee on Care, Use of Laboratory Animals, and National Institutes of Health (US). Division of Research Resources: Guide for the care and use of laboratory animals. 8th edition. National Academies Press, Washington, DC, 2011

24. Seth AK, Geringer MR, Nguyen KT, Agnew SP, Dumanian Z, Galiano RD, Leung KP, Mustoe TA and Hong SJ: Bacteriophage therapy for Staphylococcus aureus biofilm-infected wounds: A new approach to chronic wound care. Plast Reconstr Surg 131: 225-234, 2013

25. Liu D, Zhang L, Li T, Wang G, Du H, Hou H, Han L and Tang P: Negative-pressure wound therapy enhances local inflammatory responses in acute infected soft-tissue wound. Cell Biochem Biophys 70: 539-547, 2014.

26. Liu D, Li Z, Wang G, Li T, Zhang L and Tang P: Virulence analysis of Staphylococcus aureus in a rabbit model of infected full-thickness wound under negative pressure wound therapy. Antonie van Leeuwenhoek 111: 161-170, 2018.

27. Lalliss SJ, Stinner DJ, Waterman SM, Branstetter JG, Masini BD and Wenke JC: Negative pressure wound therapy reduces pseudomonas wound contamination more than Staphylococcus aureus. J Orthop Trauma 24: 598-602, 2010.

28. Kamamoto F, Lima ALM, Rezende MR, Mattar-Junior R, Leonhardt MC, Kojima KE and Santos CCD: A new low-cost negative-pressure wound therapy versus a commercially available therapy device widely used to treat complex traumatic injuries: A prospective, randomized, non-inferiority trial. Clinics (Sao Paulo) 72: 737-742, 2017.

29. Morykwas MJ, Argenta LC, Shelton-Brown EI and McGuirt W: Vacuum-assisted closure: A new method for wound control and treatment: Animal studies and basic foundation. Ann Plast Surg 38: 553-562, 1997.

30. Livak KJ and Schmittgen TD: Analysis of relative gene expression data using real-time quantitative PCR and the $2-\Delta \Delta \mathrm{Ct}$ method. Methods 25: 402-408, 2001

31. Omar A, Wright JB, Schultz G, Burrell R and Nadworny P: Microbial Biofilms and Chronic Wounds. Microorganisms 5: pii:E9, 2017.

32. Swanson EA, Freeman LJ, Seleem MN and Snyder PW: Biofilm-infected wounds in a dog. J Am Vet Med Assoc 244: 699-707, 2014.

33. Boles BR and Horswill AR: Staphylococcal biofilm disassembly. Trends Microbiol 19: 449-455, 2011

34. Licker M, Moldovan R, Hogea E, Muntean D, Horhat F, Bădiţoiu L, Rogobete A, Tirziu E and Zambori C: Microbial biofilm in human health-An updated theoretical and practical insight. Romanian J Laboratory Med 25: 9-26, 2017.

35. Li T, Zhang L, Han LI, Wang G, Yin P, Li Z, Zhang L, Guo Q, Liu D and Tang P: Early application of negative pressure wound therapy to acute wounds contaminated with Staphylococcus aureus: An effective approach to preventing biofilm formation. Exp Ther Med 11: 769-776, 2016.

36. Cornforth DM, Dees JL, Ibberson CB, Huse HK, Mathiesen IH, Kirketerp-Møller K, Wolcott RD, Rumbaugh KP, Bjarnsholt T and Whiteley M: Pseudomonas aeruginosa transcriptome during human infection 115: E5125-E5134, 2018

37. Copeland H, Newcombe J, Yamin F, Bhajri K, Mille VA, Hasaniya N, Bailey L and Razzouk AJ: Role of negative pressure wound care and hyperbaric oxygen therapy for sternal wound infections after pediatric cardiac surgery. World J Pediatr Congenit Heart Surg 9: 440-445, 2018.

38. Tahir S, Malone M, Hu H, Deva A and Vickery K: The Effect of negative pressure wound therapy with and without instillation on mature biofilms in vitro. Materials (Basel) 11: pii: E811, 2018.

39. Song J, Lays C, Vandenesch F, Benito Y, Bes M, Chu Y, Lina G, Romby P, Geissmann T and Boisset $\mathrm{S}$ : The expression of small regulatory RNAs in clinical samples reflects the different life styles of Staphylococcus aureus in colonization vs. infection. PLoS One 7: e37294, 2012.

40. Huang C,Leavitt T, Bayer LR and Orgill DP: Effect of negative pressure wound therapy on wound healing. Curr Probl Surg 51: 301-331, 2014.

41. Nie B and Yue B: Biological effects and clinical application of negative pressure wound therapy: A review. J Wound Care 25: 617-626, 2016.

42. Patil PS, Evancho-Chapman MM, Li H, Huang H, George RL, Shriver LP and Leipzig ND: Fluorinated methacrylamide chitosan hydrogel dressings enhance healing in an acute porcine wound model. PLoS One 13: e0203371-e0203371, 2018.

This work is licensed under a Creative Commons Attribution-NonCommercial-NoDerivatives 4.0 International (CC BY-NC-ND 4.0) License. 